\title{
A rare localized giant cell tumor of the tendon sheath originating from the ligamentum mucosum: A case report
}

\author{
Mehmet Arıcan, MD®, Yalçın Turhan, MD¹๑, Mehmet Gamsızkan, MD²® \\ 'Department of Orthopedics and Traumatology, Düzce University Faculty of Medicine, Düzce, Turkey \\ ${ }^{2}$ Department of Pathology, Düzce University Faculty of Medicine, Düzce, Turkey
}

Giant cell tumor of the tendon sheath (GCTTS) and synovium mainly occurs in two forms, including localized GCTTS and diffuse pigmented villonodular synovitis (PVNS). ${ }^{[1-3]}$ Giant cell tumor of the tendon sheath generally affects the small joints in the extremities and usually presents as a solitary soft tissue mass, but involvement of the major joints has recently become more prevalent. ${ }^{[4,5]}$ To date, a number of cases of GCTTS in the knee joint have been reported in the literature that describe GCTTS originating in the anterior cruciate ligament (ACL) ${ }^{[6]}$ posterior cruciate ligament $(\mathrm{PCL}){ }_{{ }^{[7]}}$ patellar tendon, ${ }^{[8]}$ and medial plica. ${ }^{[9]}$ Giant cell tumor of the tendon sheath can occur at any age but mostly affects people aged 30-50 years, particularly females. ${ }^{[3]}$ Although there are many treatment modalities for these lesions, marginal excision is the most commonly used treatment. When the lesion occurs in the knee joint, open or arthroscopic resection is used..$^{[5,10]}$ In this article, we present a rare case of GCTTS in the knee joint that had not been previously reported to originate from the ligamentum mucosum. In addition, we review the previously reported cases in the literature along with their clinical evaluation and surgical management.

\footnotetext{
Received: November 22, 2019

Accepted: December 03, 2019

Published online: March 02, 2020
}

Correspondence: Mehmet Arıcan, MD. Düzce Üniversitesi Tıp Fakültesi Ortopedi ve Travmatoloji Anabilim Dalı, 81000 Düzce, Türkiye.

E-mail: ari_can_mehmet@hotmail.com

Doi: $10.5606 /$ ehc. 2020.72323

Citation: Arıcan M, Turhan Y, Gamsızkan M. A rare localized giant cell tumor of the tendon sheath originating from the ligamentum mucosum: A case report. Jt Dis Relat Surg 2020;31(1):149-153.

\section{ABSTRACT}

Giant cell tumor of the tendon sheath (GCTTS) occurs most often in the hand and rarely in the feet, and as an extremely uncommon presentation in the knee joint. Case reports involving GCTTS in the knee joint generally describe it originating from the nearby anterior cruciate ligament, posterior cruciate ligament, patellar tendon, and medial plica. To the best of our knowledge, there are no previously reported case reports involving GCTTS originating in the ligamentum mucosum. In this article, we describe a 27 -year-old male patient who was admitted to the orthopedic emergency room with a painful locked knee. He had severe pain that was worse with activity and a decreased range of motion. Magnetic resonance imaging (MRI) indicated massive swelling and a wellcircumscribed lobulated intraarticular mass at the distal one third of the ligamentum mucosum. The mass was removed successfully with arthroscopic-assisted mini-open excision, and histological analysis subsequently diagnosed it as a localized type of GCSTT. The patient remained asymptomatic and a follow-up MRI two years after surgery did not show any recurrence of the lesion.

Keywords: Arthroscopic-assisted mini-open approach, giant cell tumor of the tendon sheath, knee, ligamentum mucosum.

\section{CASE REPORT}

A 27-year-old male patient with no significant past medical history was admitted to the orthopedic emergency department of our hospital with a painful locked right knee. There was no history of either trauma or any unusual sports injury. He reported suffering from mild pain, which was worse with activity, and a decreased range of motion for two years but denied any swelling or locking of the knee during this period. Physical examination revealed massive swelling and tenderness along the joint line and increased pain with active and passive motion of the knee. Specific knee joint tests could not be performed due to excessive pain. There were no signs of inflammation (erythrocyte sedimentation rate, C-reactive protein, and total blood count tests were normal). Plain radiography of the knee joint was also 

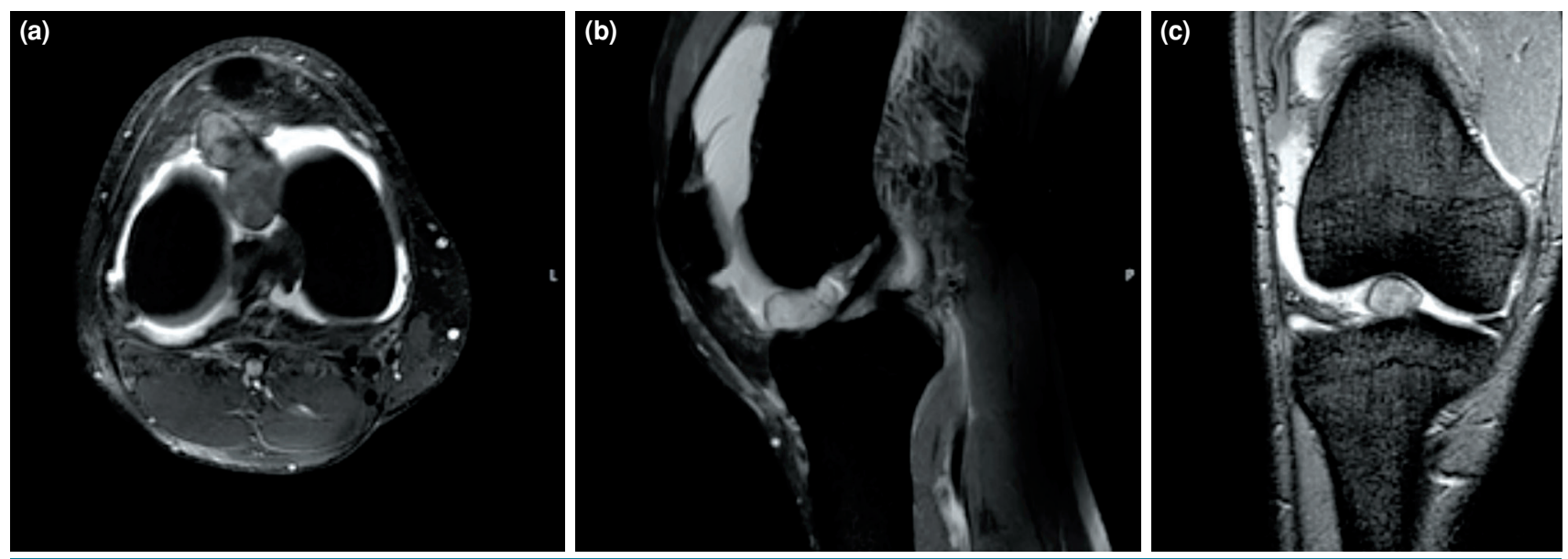

FIGURE 1. Magnetic resonance imaging indicated well circumscribed lobulated intra articular mass measuring $4 \times 2 \times 1.5 \mathrm{~cm}$. (a) transvers, (b) sagittal, (c) coronal section.

normal. Magnetic resonance imaging (MRI) revealed massive swelling and a well-circumscribed lobulated intraarticular mass, $4 \times 2 \times 1.5 \mathrm{~cm}$ in size, originating from the distal one third of the patellar side of the ligamentum mucosum (Figure 1a-c). However, no meniscal, ligamentous, or cartilaginous pathology was observed. Due to the imaging findings, GCTTS or fibroma was our primary consideration.

The arthroscopic procedure was performed under general anesthesia through standard anteromedial and anterolateral portals. After entry through the anterolateral portal, synovial fluid samples were taken for culture and Giemsa staining, which were negative. Following the sampling, the area was evaluated and the mass was identified as mainly originating from one third of the patellar side of the ligamentum mucosum (Figure 2a). Then, after entering the anteromedial portal, the mass was removed by enlarging the anteromedial portal with the help of a punch (Figure 2b). Finally, the hypertrophic and inflamed synovial tissues were debrided with an arthroscopic shaver and radiofrequency ablation.

Macroscopically, the resected mass was $4 \times 2 \times 1.5 \mathrm{~cm}$ in size, with a smooth surface, lobulated contour, and brown color (Figure 2c). Histopathologically, the mass was composed of small and round mononuclear cells
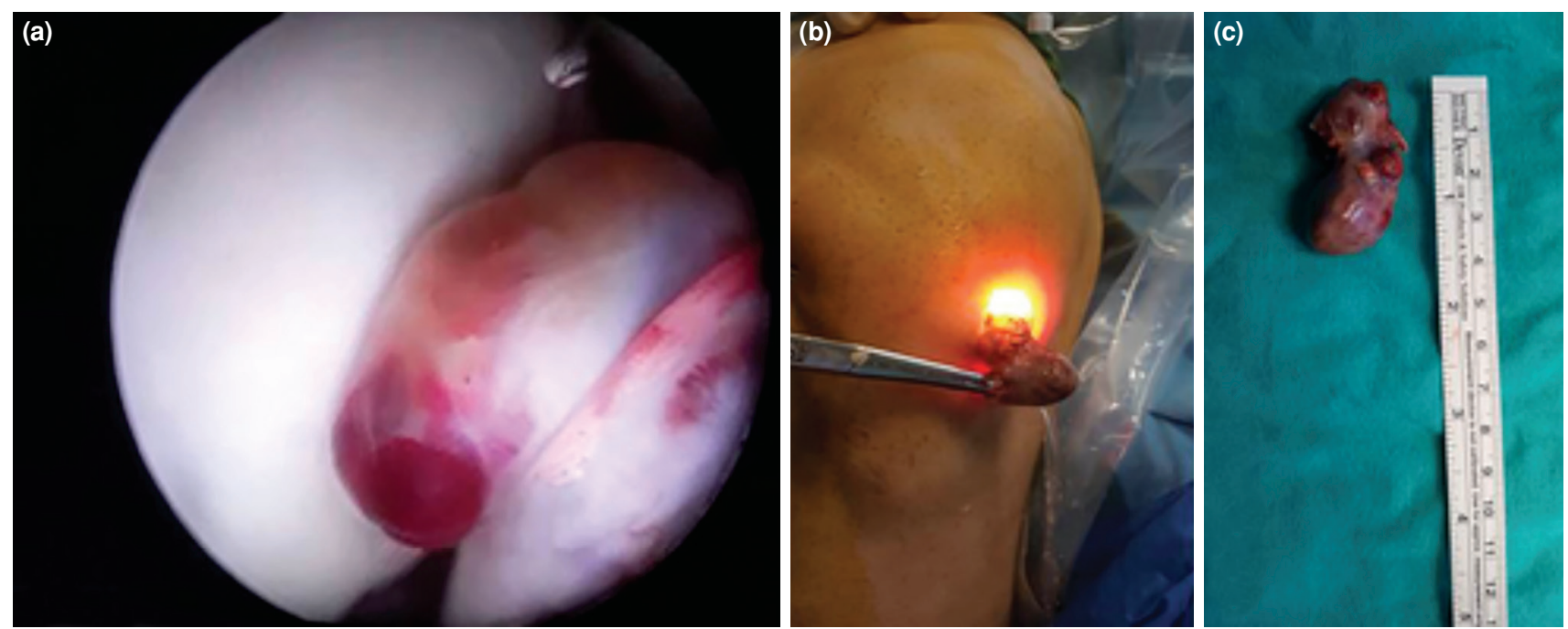

FIGURE 2. (a) Intraoperatively, on arthroscopic anterolateral portal view, well-circumscribed and lobulated mass was determined that was mainly originated from one third of patellar side of ligamentum mucosum. (b) Mass was removed by enlarging anteromedial portal with help of punch. (c) Surgical material was $4 \times 2 \times 1.5 \mathrm{~cm}$ in size, with smooth surface, lobulated contour and brown color. 


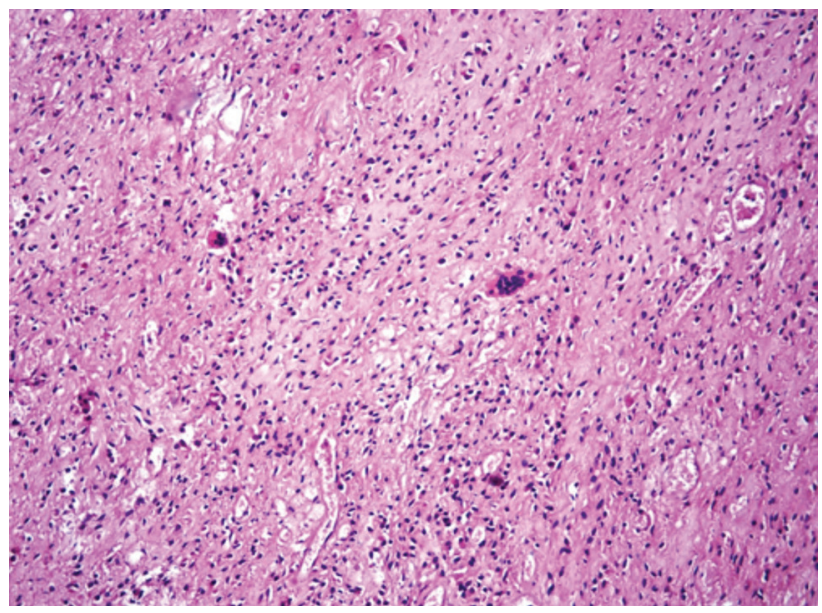

FIGURE 3. Small and round mononuclear cells and osteoclast-like giant cells $(\mathrm{H}-\mathrm{E} \times 200)$.

and osteoclast-like giant cells (Figure 3). The lesion was diagnosed as localized GCTTS. At two-year follow-up, the patient remained asymptomatic and MRI revealed no recurrence of the lesion (Figure 4a-c). A written informed written consent was obtained from the patient.

\section{DISCUSSION}

Localized giant cell tumors, also known as GCTTS, are benign nodular tumors generally found in the extremities that have originated from the synovial tissue of the joints, tendon sheath, bursa, and fibrous tissues adjacent to tendons. ${ }^{[1]}$ The etiology of GCTTS is not clear but there are many risk factors mentioned in the literature, such as trauma, infection, vascular abnormalities, lipid metabolism disorders, osteoclastic proliferation, and immune system disorders. ${ }^{[3]}$ The tumors are usually found on the tendon sheaths of the hands and feet and are extremely rare in the knee. ${ }^{[4,5,10]}$ To date, several cases of GCTTS originating in the $\mathrm{ACL}^{\left[{ }^{[6]}\right.} \mathrm{PCL}^{, 7]}$ patellar tendon, ${ }^{[8]}$ and medial plica ${ }^{[9]}$ have been reported in the literature. In this study, we present a case of GCTTS originating from the ligamentum mucosum, which, to our knowledge, has not been previously reported. There was no history of any etiological factors or medical history.

The clinical features of GCTTS in the knee joint are usually nonspecific. It can present with mild pain, swelling, a palpable mass or tenderness on the joint line, and a decreased range of motion; however, locked knee symptoms can also be present. ${ }^{[10]}$ A locked knee is a mechanical symptom that blocks full extension of the joint and results in the knee being held in flexion, which mimics a meniscal injury, loose body, ACL tear, or solitary intraarticular tumor. ${ }^{[4,11,12]}$ Dines et al. ${ }^{[4]}$ published a study involving 26 patients who underwent arthroscopic excision of localized PVNS of the knee. In this study, locked knee symptoms were detected in 10 of the 26 patients. In the present case, the patient was admitted to the orthopedic emergency room with a painful locked knee.

Histopathological examination is a definitive method for diagnosis, although physical examination and radiological imaging are also helpful preoperatively. ${ }^{[1]}$ Giant cell tumor of the tendon sheath is characterized by proliferating histiocytes and a moderately cellular appearance as sheets of rounded or polygonal cells. In addition, hemosiderin (brown color) may be present but typically less than is
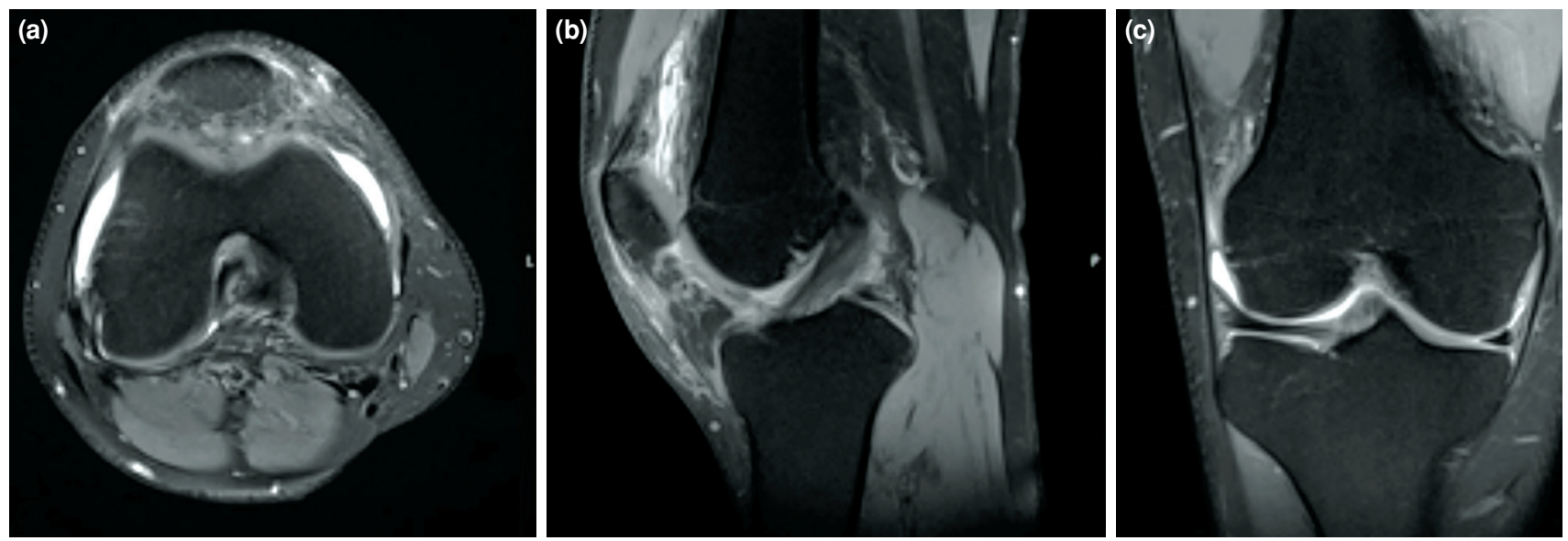

FIGURE 4. Magnetic resonance imaging two years after surgery showed no recurrence of lesion (a) transvers, (b) sagittal, (c) coronal section. 
seen in PVNS, and multinucleated giant cells are common. ${ }^{[2,3,13]}$ Differential diagnosis includes ganglion cyst, PVNS, or fibromas. ${ }^{[1,13]}$ Pigmented villonodular synovitis is histologically identical with GCTTS but it most commonly involves the whole synovium in larger joints and pronounced hemosiderin, which produces a characteristic signal on MRI. ${ }^{[14]}$ Giant cell tumor of the tendon sheath and ganglion cysts can be distinguished by ultrasound; GCTTS is solid, nodular, and has internal vascularity while a ganglion cyst has a cystic component with well-defined borders and no internal vascularity. ${ }^{[4,15]}$ Fibromas and GCTTS have distinct features on MRI, including differences in lesion morphology and intensity patterns. In addition, GCTTS is more uniform and has a mixed cell population that can help to distinguish it from fibromas. ${ }^{[14]}$ The primary clinical diagnosis in our patient was meniscal injury or ACL tear. After radiological evaluation with MRI, we suspected GCTTS or fibroma but after arthroscopic excision with arthroscopy and histopathological examination, a final diagnosis of localized tenosynovial GCTTS was established.

Although there are many treatment modalities for GCTTS, marginal excision is the most commonly used. ${ }^{[2]}$ Arthroscopic or open excision of GCTTS in the knee joint has been described in previous studies. ${ }^{[4,5]}$ Open surgery allows adequate marginal excision of surrounding tissues that may have been invaded by the tumor and, therefore, exhibits a decreased risk of local recurrence when compared with arthroscopic surgery. ${ }^{[1]}$ In contrast, Dines et al. ${ }^{[4]}$ evaluated the longterm follow-up of surgically treated localized PVNS of the knee after open and arthroscopic surgery. The study examined 14 GCTTS patients who underwent open excision and 12 who underwent arthroscopic excision. None of the 12 patients who underwent arthroscopic surgery required a second surgery. Recurrence is the most frequent complication for both localized (GCTTS) and diffuse (diffuse PVNS) tumors. ${ }^{[1-3]}$ The local recurrence rate has been reported to be between $4-45 \%$ in most cases; however, only a few studies have been conducted. ${ }^{[4,5,10]}$ Loriaut et al. ${ }^{[10]}$ published a study involving 30 patients with localized PVNS of the knee who were treated arthroscopically and found that only four patients had recurrence at a mean 75-month follow-up. In another study, Zhang et al. ${ }^{[5]}$ reported no recurrence after arthroscopic excision at a mean 15-year follow-up, and they concluded that arthroscopic removal of GCTTS is an effective treatment. In our patient, marginal excision of the tumor was performed arthroscopically and there were no signs of recurrence at the two-year follow-up.
To the best of our knowledge, the presented case of GCTTS originating from the ligamentum mucosum is the first to be reported in the literature. The rarity of the presented case suggests that intraarticular GCTTS should be considered in the differential diagnosis of a painful locked knee, and arthroscopy assisted mini-open approach is a successful treatment with a low rate of recurrence.

\section{Declaration of conflicting interests}

The authors declared no conflicts of interest with respect to the authorship and/or publication of this article.

\section{Funding}

The authors received no financial support for the research and/or authorship of this article.

\section{REFERENCES}

1. van der Heijden L, Gibbons CL, Dijkstra PD, Kroep JR, van Rijswijk CS, Nout RA, et al. The management of diffuse-type giant cell tumour (pigmented villonodular synovitis) and giant cell tumour of tendon sheath (nodular tenosynovitis). J Bone Joint Surg [Br] 2012;94:882-8.

2. Kant KS, Manav AK, Kumar R, Abhinav, Sinha VK, Sharma A. Giant cell tumour of tendon sheath and synovial membrane: A review of 26 cases. J Clin Orthop Trauma 2017;8(Suppl 2):S96-S9.

3. Monaghan H, Salter DM, Al-Nafussi A. Giant cell tumour of tendon sheath (localised nodular tenosynovitis): clinicopathological features of 71 cases. J Clin Pathol 2001;54:404-7.

4. Dines JS, DeBerardino TM, Wells JL, Dodson CC, Shindle M, DiCarlo EF, et al. Long-term follow-up of surgically treated localized pigmented villonodular synovitis of the knee. Arthroscopy 2007;23:930-7.

5. Zhang WG, Wang LD, Li J, Zhang YF, Liu Y, Wang FS. Arthroscopic treatment of the giant cell tumor of tendon sheath in knee joint. Zhonghua Wai Ke Za Zhi 2006;44:258-9.

6. Otsuka Y, Mizuta H, Nakamura E, Kudo S, Inoue S, Takagi $\mathrm{K}$. Tenosynovial giant-cell tumor arising from the anterior cruciate ligament of the knee. Arthroscopy 1996;12:496-9.

7. Camillieri G, Di Sanzo V, Ferretti M, Calderaro C, Calvisi V. Intra-articular tenosynovial giant cell tumor arising from the posterior cruciate ligament. Orthopedics 2012;35:e1116-8.

8. Khanduja V, Dannawi Z, Ng L, El-Zebdeh M. Giant-cell tumor of the tendon sheath arising from the patellar tendon. J Knee Surg 2004;17:28-9.

9. Kim YM, Joo YB. Localized nodular tenosynovitis originated near the medial plicae. Knee Surg Relat Res 2014;26:52-5.

10. Loriaut P, Djian P, Boyer T, Bonvarlet JP, Delin C, Makridis KG. Arthroscopic treatment of localized pigmented villonodular synovitis of the knee. Knee Surg Sports Traumatol Arthrosc 2012;20:1550-3.

11. Snoj Ž, Pižem J, Salapura V. Sudden onset of severe anterior knee pain and knee locking during sleep. Skeletal Radiol 2016;45:407-8.

12. Gülenç B, Kuyucu E, Yalçin S, Çakir A, Bülbül AM. Arthroscopic excision of tendinous giant cell tumors causing locking in the knee joint. Acta Chir Orthop Traumatol Cech 2018;85:109-12. 
13. Sezgin EA, Atik OŞ. Tumors and tumor-like lesions of infrapatellar fat pad and surrounding tissues: A review of the literature. Eklem Hastalik Cerrahisi 2018;29:58-62.

14. Ge Y, Guo G, You Y, Li Y, Xuan Y, Jin ZW, et al. Magnetic resonance imaging features of fibromas and giant cell tumors of the tendon sheath: differential diagnosis. Eur Radiol 2019;29:3441-9.

15. Wang Y, Tang J, Luo Y. The value of sonography in diagnosing giant cell tumors of the tendon sheath. J Ultrasound Med 2007;26:1333-40. 